\title{
Changing the wolf from outside: how microbiota trigger systemic lupus erythematosus
}

\author{
Isabelle Peene, ${ }^{1,2}$ Dirk Elewaut ${ }^{\oplus 1,2}$
}

Systemic lupus erythematosus (SLE) is a prototypic autoimmune disorder affecting multiple organ systems including skin, joint and several internal organs such as kidney, heart or lung. A hallmark of this disease is the development of circulating antibodies against nuclear antigens and immune complex formation. The bestknown antibodies are directed against native DNA and are prognostic for development of lupus nephritis. The processes resulting to the cascade of events culminating in the clinical features of SLE are only partially understood and involve among others, defective clearance of apoptotic cells and enhanced DNA damage to ultraviolet irradiation, and netosis $^{1} 2$ (figure 1). It has also been suggested for a long time that certain microbial triggers could play a role in the onset of SLE, supported by early reports that antibody responses to nucleic acids/ nucleoproteins were found in association with certain bacterial infections. More recently, more definitive proof of concept studies using germ-free and reconstituted mice highlighted that intestinal microbiota are able to drive development of systemic autoantibody responses. ${ }^{3} 4$ However, whether similar processes underlie development of lupus nephritis and anti-DNA antibodies was unclear.

In Annals of Rheumatic Diseases, Azzouz et $a l^{5}$ describe the results of an in-depth analysis of the relationship between intestinal microbiota, development of anti-DNA antibodies and their link to development of lupus nephritis. 16S rRNA sequencing was conducted on faecal samples from a discovery SLE cohort of 61 patients. In this cohort, the investigators described a clear evidence of dysbiosis with the fivefold overabundance of Ruminococcus gnavus (RG) versus controls being the most significant

${ }^{1}$ Unit Molecular Immunology and Inflammation, Inflammation Research Center, VIB-Ghent University, Ghent, Belgium

${ }^{2}$ Department of Rheumatology, University Hospital Ghent, Gent, Belgium

Correspondence to Dr Dirk Elewaut, Unit Molecular Immunology and Inflammation, Inflammation Research Center, VIB-Ghent University, Ghent 9052, Belgium; dirk.elewaut@ugent.be finding. RG is an anaerobic gram-positive taxon in the Fimicutes phylum and Lachnnospiraceae family and has been previously associated with ankylosing spondylitis (AS) and inflammatory bowel diseases (IBDs). ${ }^{6}$ Intriguingly, its relative abundance correlated with lupus disease activity. Outgrowth of Ruminococcus was associated with reciprocal reductions of intestinal species such as Bacteroides uniformis. In addition to this, the investigators also revealed some evidence for impaired intestinal barrier integrity in a significant proportion of the patients with SLE. This could favour loss of systemic tolerance. To test the impact of overabundance of RG in patients with SLE, serum immunoglobulin (Ig)A and IgG antibody responses were measured. They were not only increased in SLE versus controls but the level of IgG anti-RG2 antibodies correlated with the faecal abundance of RG as well as the SLE Disease Activity Index score, a validated measure of disease activity. Based on these impressive correlations, the investigators evaluated the association between anti-RG and anti-native DNA antibodies, which are commonly found in patients with lupus nephritis. A number of observations listed below revealed a striking and selective association between anti-RG IgG antibodies and occurrence of anti-DNA antibodies, including correlation with $\operatorname{IgG}$ reactivity against human glomerular extracts, and inverse association with C3 and C4 levels indicative of complement activation typically observed in immune-complex mediated diseases such as lupus nephritis. The investigators then sought to unravel the molecular nature of this observed association and found that RG2 but not RG1 antigens cross-react with lupus anti-dsDNA antibodies. Using a hydrophobic interaction chromatography-based strategy to characterise cell wall moeities, fractions of the RG2 strain were obtained and tested by nuclear magnetic resonance and mass spectrometry analysis and tested for immune reactivity using SLE sera. The lipoglycan 3 cell wall expressed the immunodominant epitopes commonly recognised by SLE sera.
The association with RG2 antibodies and anti-native DNA autoantibodies could be replicated in two small cohorts of, respectively, 27 patients with SLE and 17 patients with active lupus nephritis but they were absent in patients with primary membranous glomuleronephritis and antineutrophil cytoplasmic antibody associated vasculitis suggesting their relative specificity. In addition, the association with anti-RG2 antibodies was not found with other SLE autoantibodies such as anti-Ro. Collectively, the data shown by Azzouz et al reveal a novel and intriguing paradigm that SLE may be triggered or augmented through a molecular mimicry process between a lipoglycan expressed on the cell wall of intestinal RG2 and native DNA molecules (figure 1). However, whether these findings reflect causal associations is yet unclear. Some indirect evidence suggest that RG may foster barrier integrity loss as the Lachnnospiraceae family fill a special niche and degrade complex polysaccharides such as the intestinal mucus layer. On the other hand, expansion of RG strains has also been reported in both IBD and AS, ${ }^{6}$ yet whether they are identical as in SLE remains unclear. It is also unknown whether development of anti-RG antibodies is a SLE-specific trait. In this regard, larger groups of controls including IBD, AS, spondyloarthritis $(\mathrm{SpA})$ need to be tested. In addition, patients with SLE and Sjogren's syndrome (SS), two closely related autoimmune diseases, share a common restricted gut microbiota diversity, but their oral microbiota are distinct. ${ }^{7}$ Knowledge of the gut microbiota diversity in relation to an autoantibody status shared by different diseases (for instance, as for antiRo52 in SLE, SS, systemic sclerosis and idiopathic inflammatory myopathy) might further untangle this conundrum. An earlier study reported that sera from patients with SLE with antiRo60 antibodies immunoprecipitate orthologs Ro60 derived from commensal bacteria. ${ }^{8}$ In contrast to anti-DNA antibodies, this autoreactivity remains stable in the long term suggesting that it may be less influenced by microbiota alterations over time. Further research should elucidate whether the fluctuations of anti-DNA antibodies in patients run parallel to shifts in the diversity of gut microbiota. Furthermore, most definitive cause-effect studies such as gnotobiotic mouse models reconstituted with RG versus control microbiota on SLE features still need to be reported, but given the earlier reported association between intestinal microbiota, segmented filamentous bacteria (a strong inducer of Th17 responses) and development of antinuclear antibodies, it is tempting to assume that the 


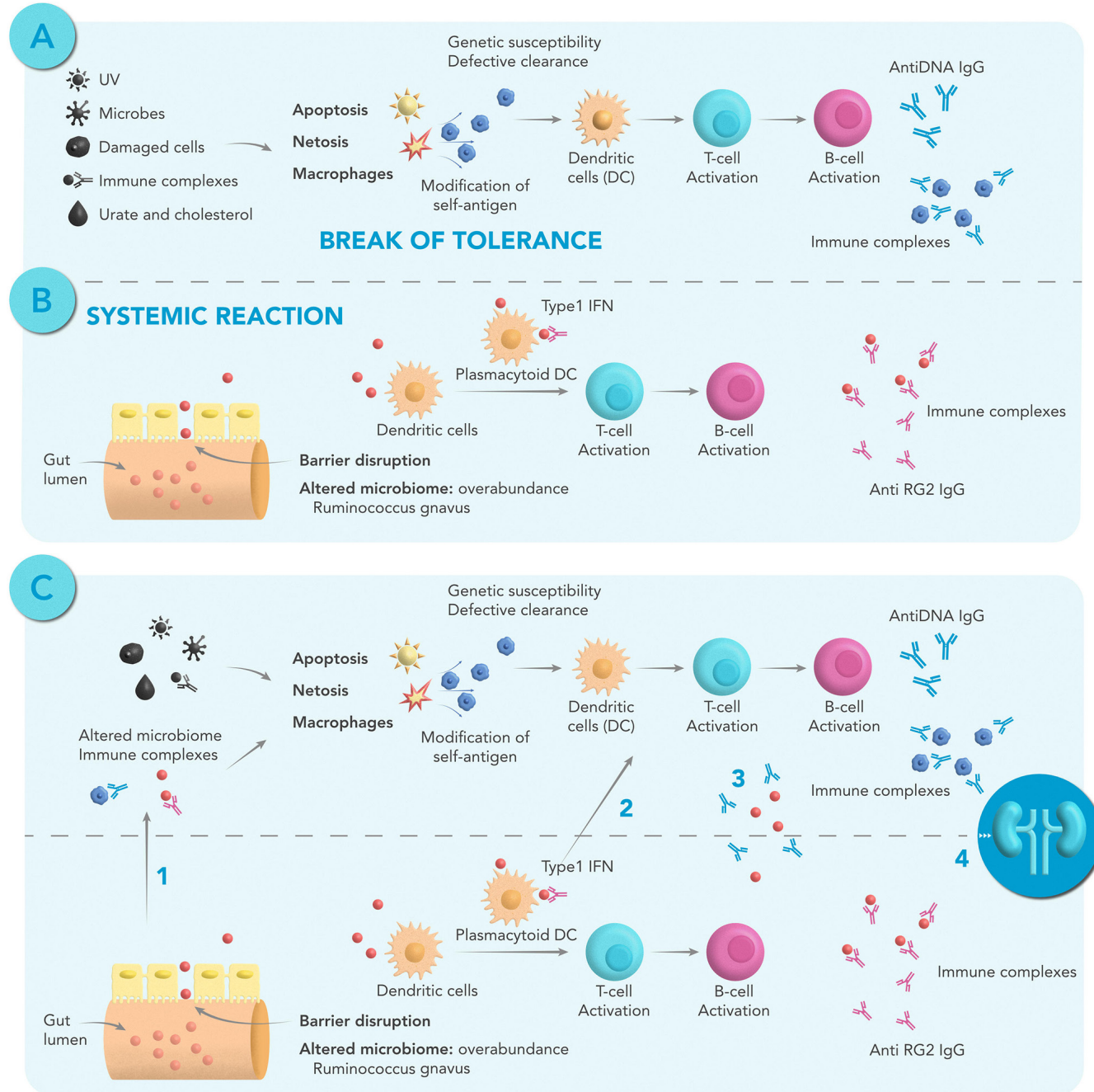

Figure 1 Integrating intestinal microbiota into systemic lupus erythematosus (SLE) pathogenesis. (A) Exposure to various sterile and/or infectious stimuli can lead to apoptosis or NETosis contributing to release of modified self-antigen (DNA). Genetic susceptibility and/or defective clearance results in presentation of these modified self-antigens to T cells followed by B cell activation and eventually production of anti-DNA antibodies, wellknown for their association with lupus nephritis. (B) Dysbiosis in patients with SLE with fivefold overabundance of Ruminococcus gnavus (RG) leads to barrier disruption and loss of systemic tolerance with subsequent development of anti-RG antibodies mainly directed towards the fragment RG2 (anti-RG2 antibodies). RG2-containing pools included moieties that were potent in vitro activators via toll-like receptor 2 (TLR2) (previously been implicated in lupus pathogenesis), illustrated by Azzouz et $a^{5}$ by elevated serum interferon alpha 2. (C) SLE may be triggered or augmented through intestinal dysbiosis and a molecular mimicry process between a lipoglycan expressed on the cell wall of RG and native DNA molecules (1) Loss of systemic tolerance due to over abundance of RG and/or the formation of immune complexes may lead to augmented stimulation of innate immunity and subsequent enhanced presentation of self antigen followed by production of anti DNA antibodies. (2) Type1 interferon has a critical role in SLE pathogenesis. (3) Anti-DNA antibodies cross-react with RG2, more specifically the lipoglycan 3 cell wall expressing the immunodominant epitopes. Cross-reactivity of lupus autoreactive B cells to leaked stimulatory RG bacterial components, including TLR ligands(s), may contribute to the initiation and/or flares of SLE. (4) Kidney injury by direct antibody binding or by deposition of immune complexes.

current associations reflect a true causal association in genetically predisposed individuals (figure 1). Alternatively, the association could reflect a lowered threshold for systemic autoantibody development. Collectively, the data presented here reflect the relevance of an emerging theme that epithelial surfaces constitute a gateway to a myriad of rheumatic diseases including rheumatoid arthritis, SpA including AS and psoriatic arthritis, now joined by SLE.

Correction notice This article has been corrected since it published Online First. The figure legend has been amended.
Handling editor Josef S Smolen

Contributors IP and DE wrote jointly this editorial. Figure concept was made by IP.

Funding This study was supported by Research Council of Ghent University and Fund for Scientific Research Flanders.

Competing interests None declared.

Patient consent for publication Not required.

Provenance and peer review Commissioned; externally peer reviewed.

(c) Author(s) (or their employer(s)) 2019. No commercial re-use. See rights and permissions. Published by BMJ.

\section{Check for updates}

To cite Peene I, Elewaut D. Ann Rheum Dis 2019;78:867-869.

Received 27 March 2019

Revised 31 March 2019

Accepted 1 April 2019

\section{Linked}

- http://dx.doi.org/10.1136/annrheumdis-2018214856

Ann Rheum Dis 2019;78:867-869. 
doi:10.1136/annrheumdis-2019-215221

\section{REFERENCES}

1 Gupta S, Kaplan MJ. The role of neutrophils and NETosis in autoimmune and renal diseases. Nat Rev Nephrol 2016;12:402-13.

2 Frangou E, Chrysanthopoulou A, Mitsios A, et al. REDD1/autophagy pathway promotes thromboinflammation and fibrosis in human systemic lupus erythematosus (SLE) through nets decorated with tissue factor (TF) and interleukin-17A (IL-17A). Ann Rheum Dis 2019;78:238-48.

3 Van Praet JT, Donovan E, Vanassche I, et al. Commensal microbiota influence systemic autoimmune responses. Embo J 2015;34:466-74.

4 Van de Wiele T, Van Praet JT, Marzorati M, et al. How the microbiota shapes rheumatic diseases. Nat Rev Rheumatol 2016:12:398-411.

5 Azzouz D, Omarbekova A, Heguy A, et al. Lupus nephritis is linked to disease-activity associated expansions and immunity to a gut commensal. Ann Rheum Dis 2019;78:947-56.
6 Breban M, Tap J, Leboime A, et al. Faecal microbiota study reveals specific dysbiosis in spondyloarthritis. Ann Rheum Dis 2017:76:1614-22.

7 van der Meulen TA, Harmsen HJM, Vila AV, et al. Shared gut, but distinct oral microbiota composition in primary Sjögren's syndrome and systemic lupus erythematosus. J Autoimmun 2019;97:77-87.

8 Greiling TM, Dehner C, Chen X, et al. Commensal orthologs of the human autoantigen Ro60 as triggers of autoimmunity in lupus. Sci Trans/ Med 2018;10:pii: eaan2306. 